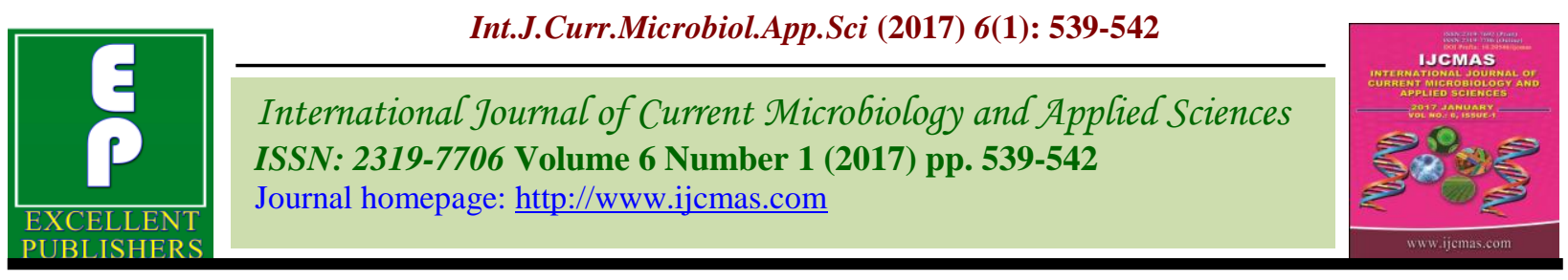

Letter to Editor

http://dx.doi.org/10.20546/ijcmas.2017.601.066

\title{
MDR-TB and XDR-TB Surveillance Highlights Need for Rapid Detection of Drug-Resistance in Mycobacterium Tuberculosis Infections
}

\author{
Priyanka Soni Gupta ${ }^{1 *}$, Tarun Patni ${ }^{2}$, Ankur Gupta ${ }^{3}$, Rakesh C Gupta ${ }^{4}$, and \\ Payal Mathur ${ }^{5}$
}

\begin{abstract}
${ }^{1}$ Department of Microbiology, ${ }^{2}$ State TB Demonstration Centre, ${ }^{3,4}$ Department of Pulmonary Diseases, ${ }^{5}$ Senior Demonstrator Department of Microbiology Jawahar Lal Nehru Medical College and Associated group of Hospitals, Ajmer, Rajasthan, India
\end{abstract}

*Corresponding author:

Drug-resistance is a growing threat to global efforts in controlling and managing TB infections. Drug-susceptible TB is curable but drug misuse and poor patient compliance leads to multidrug resistance to the first line of TB antibiotics, rifampicin and isoniazid. The second line of treatment is less potent and has severe side effects, takes longer to cure the disease, and has poor success rates (Gandhi et al., 2010). However, continued drug misuse can result in extensive resistance to second line drugs, drastically limiting further treatment options.

This is concerning because multidrug resistant (MDR) and extensively drug resistant (XDR) TB can be transmitted to uninfected individuals: MDR-TB in new TB cases reached up to 35\% in 2013 (World Health Organization, 2014). Poor prognosis of MDR$\mathrm{TB}$ and XDR-TB infections underlines the need for aggressive management and control measures to stem the rise of drug-resistant TB.

India is recognized as one of the high TB and MDR-TB burden countries. The Directly
Observed Treatment, Short-course (DOTS) program improved TB/MDR-TB diagnosis and patient compliance and decreased TB incidence and mortality in India, but drugresistant $\mathrm{TB}$ continues to pose a challenge. Timely diagnosis of MDR-TB and initiation of second-line of treatment has been a major hurdle in tackling the spread and treatment of drug-resistant TB. Long turnaround times in MDR-TB diagnosis (3-4 months for solid culture drug susceptibility testing) leads to poor treatment outcomes, high mortality rates in patients, as well as increased risk of transmitting drug-resistant TB to uninfected individuals ( Raizada et al., 2014). In contrast, rapid molecular tests line probe assays (LPA) can detect drug-resistance to first-line drugs within 8 hours, significantly improving the prognosis of patients with MDR-TB.

In this study, we analyze the MDR-TB trends in northwestern India and identify the secondline drugs to which resistance is developing in the region. Up until 2014, all new TB patients are treated with first-line drugs for 2 months, and if the sputum is positive for the bacterium after this period, the patients are tested for drug-resistance by LPA, sometimes in 
combination with the standard drug susceptibility testing (DST). If drug-resistance is confirmed, treatment with second-line drugs is initiated.

To understand the impact of rapid diagnosis of drug-resistance on MDR-TB trends, we collected statistics on LPA performed at the State TB Demonstration Centre (STDC) at Ajmer, Rajasthan: a government-run center for testing multidrug resistance in MDRsuspect $\mathrm{TB}$ cases as per national guidelines for clinical management of MDR-TB. Patients with a confirmed MDR-TB diagnosis are considered XDR-suspects and are tested for resistance to second-line drugs. LPA for second-line drugs is available but not recommended due to low sensitivity of the test(4). Thus, for XDR-suspect cases, sputum specimens are forwarded to the National Tuberculosis Institute (NTI), Bangalore, for culture-based DST. NTI reports resistance to four second-line drugs: Capreomycin, Kanamycin, Amikacin, and Ofloxacin. Cumulative statistics on MDR- and XDRresistance were obtained from the STDC, without any identifying patient information.

A faster turnaround time led to more samples being tested for drug-resistance via LPA compared to DST, and a higher number of samples with resistance to either or both of the first-line drugs were detected (Figure 1).

The regional incidence of MDR-TB is found to be gradually decreasing(Figure 1), in agreement with there cent trends in global MDR-TB incidence (Cohen et al., 2014). MDR-TB incidence in January-September 2014 is less than half of the previous calendar year, suggesting that this decline continued in the year 2014 as well.

Fig.1 Drug-resistant TB in northwestern India
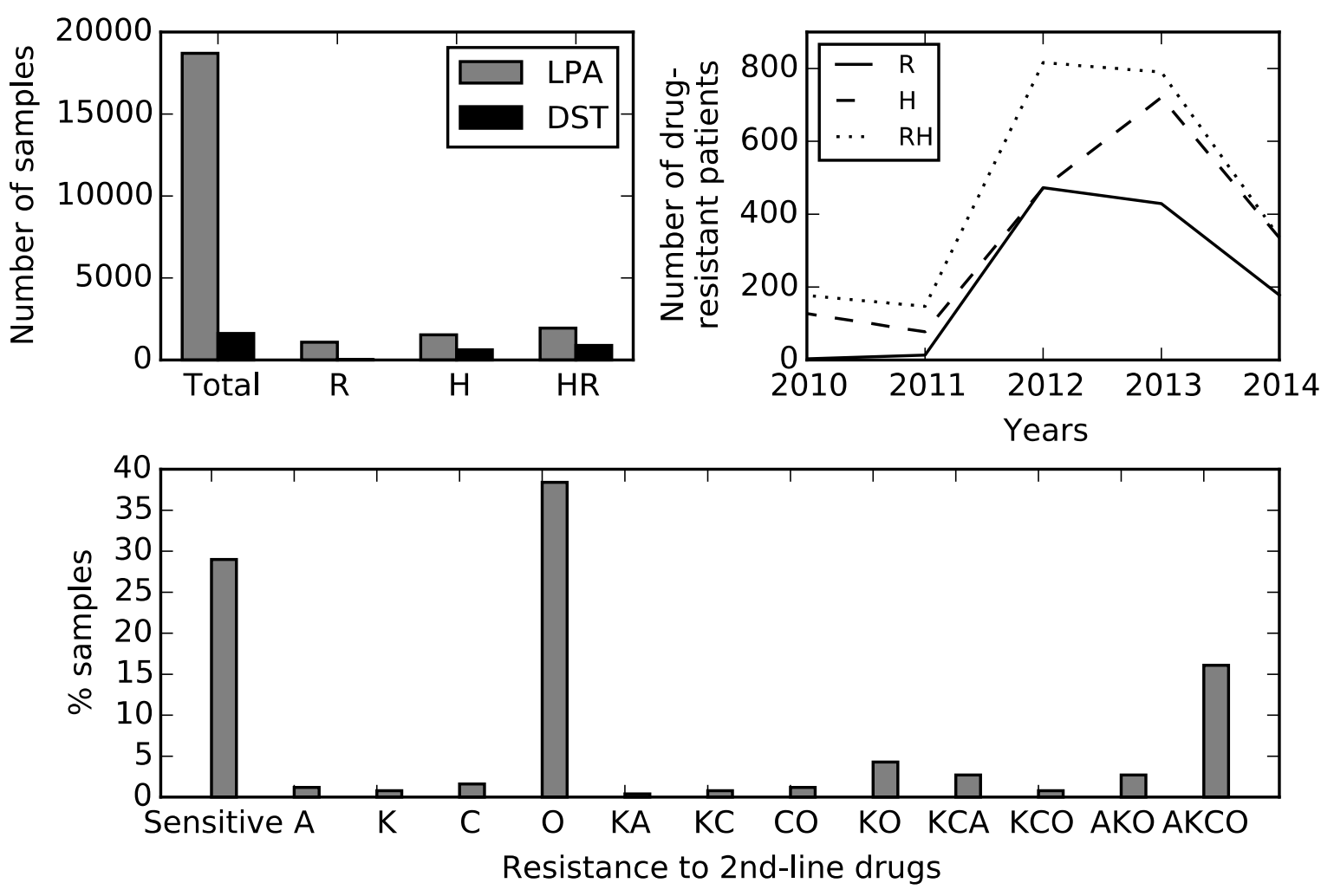
Drug susceptibility testing of sputum samples from XDR-suspects showed that the bacterium is developing resistance to some second-line drugs more than the others.

Ofloxacin resistance was widespread, and so was the resistance to drug-cocktails that contained Ofloxacin. Of the 255 clinical samples up until March 2014, 29\% were sensitive to the four drugs tested, $42 \%$ were resistant to a single second-line drug, $6.7 \%$ were resistant to two drugs, $6.3 \%$ were resistant to three drugs, and $16 \%$ of samples were resistant to all four second-line drugs (Figure 1).

Top left plot shows the number of samples tested for drug-resistance in 2012-2014 using Line Probe Assay (LPA) and Drug Susceptibility Testing (DST). R: samples resistant to rifampicin, $\mathrm{H}$ : samples resistant to isoniazid, HR: samples resistant to both. Topright plot shows the decrease in incidence of MDR-TB in Rajasthan over the years. LPA was introduced in 2010, explaining the lower incidence in 2010-2011.

Note that for year 2014, data is from JanuarySeptember ( 9 months), yet the MDR-TB incidence is less than half of the entire previous year. Bottom plot shows resistance to second-line TB antibiotics in Rajasthan in 2012-2014.

A: Amikacin, K: Kanamycin, C: Capreomycin, O: Ofloxacin. Resistance to combinations of these drugs is also shown, for example, "KA" implies resistance to Kanamycin and Amikacin, and "KCA" implies resistance to Kanamycin, Capreomycin, and Amikacin. Resistance to Ofloxacin, and drug-combinations containing Ofloxacin, appears to be prevalent in the region.

Faster detection of drug-resistance and timely initiation of second-line treatment can lead to decline in MDR-TB incidence. We suspect that this decline will accelerate if all new TB cases are tested for drug-resistance instead of administering first-line drugs for 2 months without testing for resistance. Although we do not have enough clinical data to identify trends for XDR-TB in the region, resistance to second-line drug Ofloxacin is particularly alarming, suggesting that this drug should be used with caution for XDR-TB patients in the region.

While stronger biomarker candidates are needed to develop sensitive LPA for XDR$\mathrm{TB}$, regional drug-susceptibility testing statistics can be used to guide local clinical management of extensively drug-resistant TB. However, since first-line drugs are most potent and successful in treating TB infections, further improvement in clinical management and patient compliance in susceptible TB cases is critical for the systematic fight against global TB epidemic.

\section{References}

Cohen, T., Jenkins, H.E., Lu, C., McLaughlin, M., Floyd, K., Zignol, M. 2014. On the spread and control of MDR-TB epidemics: An examination of trends in anti-tuberculosis drug resistance surveillance data. Drug Resistance Updates, 17(4-6): 105-23.

Gandhi, N.R., Nunn, P., Dheda, K., et al. 2010. Multidrug-resistant and extensively drug-resistant tuberculosis: a threat to global control of tuberculosis. Lancet, 375(9728): 1830-43.

Raizada, N., Sachdeva, K.S., Chauhan, D.S., et al. 2014. A Multi-Site Validation in India of the Line Probe Assay for the Rapid Diagnosis of Multi-Drug Resistant Tuberculosis Directly from Sputum Specimens. Plos One, 9(2). 
WHO Expert Group Meeting Report. 2013. World Health Organization, 2014, Global http://apps.who.int/iris/bitstream/10665/7 8099/1/WHO_HTM_TB_2013.01.eng.pd Tuberculosis Report.

f.

\section{How to cite this article:}

Priyanka Soni Gupta, Tarun Patni, Ankur Gupta and Rakesh C Gupta. 2017. MDR-TB and XDR-TB Surveillance Highlights Need for Rapid Detection of Drug-Resistance in Mycobacterium Tuberculosis Infections. Int.J.Curr.Microbiol.App.Sci. 6(1): 539-542. doi: http://dx.doi.org/10.20546/ijcmas.2017.601.066 